\title{
Hematoma de Músculo Iliopsoas na Vigência de Tratamento com Varfarina
}

\author{
Iliopsoas Muscle Hematoma During Treatment with Warfarin
}

\author{
Gabriel Zago ${ }^{1}$, Marcelo Campos Appel-da-Silva', Luiz Claudio Danzmann² \\ Hospital Nossa Senhora da Conceição, Porto Alegre, RS'; Hospital São Lucas da PUCRS², Porto Alegre, RS - Brasil
}

A varfarina é droga amplamente utilizada na prevenção de fenômenos tromboembólicos e o conhecimento de seus efeitos adversos faz-se necessário para o acompanhamento dos pacientes. Embora o desenvolvimento de discrasias sanguíneas seja complicação potencial nesses pacientes, a ocorrência de sangramento retroperitoneal é rara. Este artigo discute o caso de um paciente que evoluiu com hematoma do músculo iliopsoas durante tratamento com a referida droga, pós-implante de prótese aórtica metálica, com quadro clínico envolvendo importantes diagnósticos diferenciais.
Warfarin is a widely used drug for the prevention of thromboembolic events. Knowledge of its adverse effects is necessary for patient follow-up. Although the development of blood dyscrasias is a potential complication in these patients, retroperitoneal bleeding is rare. This article reports the case of a patient who developed iliopsoas muscle hematoma during treatment with warfarin after implantation of a metallic prosthetic aortic valve. The clinical manifestations involved important differential diagnoses.

\section{Introdução}

A anticoagulação oral contínuada está indicada em diversas situações clínicas, e a varfafina é a droga mais amplamente utilizada para prevenção e tratamento de fenômenos tromboembólicos e na redução do risco de morte decorrente de infarto agudo do miocárdio (IAM) e suas complicações ${ }^{1}$. Em pacientes hospitalizados, a administração das medicações e a adequação da dose, por meio de exames laboratoriais, permitem o controle e a segurança da meta terapêutica a ser atingida. Sob o ponto de vista de acompanhamento ambulatorial, o seguimento desses pacientes nem sempre é regular. Com a perda do acompanhamento e o desconhecimento dos níveis do tempo de protrombina (TP) e da razão normalizada internacional $(\mathrm{RNI})$, cresce o risco de efeitos adversos, dentre os quais, a hemorragia intracraniana ou retroperitoneal são as mais importantes e potencialmente fatais ${ }^{2}$.

A ocorrência de hematoma de músculo iliopsoas na vigência de tratamento anticoagulante é rara. Sua apresentação é usualmente unilateral, com sintomatologia relacionada à compressão do plexo nervoso do membro inferior e com diagnóstico diferencial abrangendo diversas áreas clínicas ${ }^{3}$.

\section{Palavras-chave}

Coagulação sanguínea, varfarina, hematoma, espaço retroperitoneal, músculos psoas.

Correspondência: Marcelo Campos Appel da Silva •

Rua Com. Caminha, 250 /902 - M Vento - 90430-030 - Porto Alegre,

RS - Brasil

E-mail: marceloappel@yahoo.com.br

Artigo recebido em 01/02/09; revisado recebido em 15/05/09;

aceito em 15/05/09.
O presente artigo tem por objetivo relatar o caso de um paciente que desenvolveu complicação bastante incomum e de difícil diagnóstico associada ao uso de anticoagulante oral para prevenção de acidentes tromboembólicos em portador de prótese valvar mecânica em posição aórtica.

\section{Relato do caso}

Um paciente do sexo masculino, 68 anos, branco, agricultor, procedente de Canoas, Rio Grande do Sul, procurou atendimento na emergência do Hospital Universitário da Ulbra (Canoas-RS) com queixa de dor, parestesia pré-tibial e paresia no membro inferior esquerdo, iniciadas há 3 dias. À chegada, o paciente apresentava bom estado geral, estava lúcido, com mucosas úmidas e coradas, pressão arterial de 130/80 mmHg, frequência cardíaca de 110 bpm e afebril. Durante a inspeção, verificaram-se hematomas nas coxas e no abdome. Constatou-se ausculta cardíaca com ritmo regular e sopro telessistólico audível em todo o precórdio. O exame abdominal não apresentou nenhuma particularidade. No exame neurológico, observaram-se monoparesia crural de grau 3, reflexos simétricos e sinal cutâneo-plantar negativo nos dois membros inferiores.

Inquerido sobre sua história médica pregressa, o paciente referiu diagnóstico de hipertensão há 15 anos, em tratamento com captopril 25 mg, 8/8 horas, e afirmou ter realizado cirurgia para troca da valva aórtica por prótese mecânica St. Jude há 2 anos. Desde a alta hospitalar, o paciente utilizava varfarina sódica $5 \mathrm{mg} /$ dia. Há 14 dias, seu cardiologista indicou um aumento da dose para 7,5 mg/dia. Na última semana, sofreu uma queda que resultou em trauma lombar leve, sem repercussão em suas atividades e sem queixa de dor.

Após a avaliação inicial na emergência do Hospital Universitário da Ulbra, solicitaram-se tomografia computadorizada (TC) de crânio sem contraste, 
eletrocardiograma de repouso, hemograma, provas de coagulação, função renal, eletrólitos, LDH e bilirrubinas para investigação do quadro clínico.

Os resultados dos exames mostraram TC de crânio sem evidências de lesão hemorrágica ou isquêmica aguda. O eletrocardiograma apresentou alterações compatíveis com hipertrofia de ventrículo esquerdo. Nos exames laboratoriais, verificaram-se função renal e eletrólitos normais, leucograma sem sinais sugestivos de infecção, 231.000 plaquetas, tempo de protrombina com atividade inferior a 10\%, RNI superior a 5, tempo de tromboplastina parcial ativada (TTPa) $=82,4$ segundos, desidrogenase lática $(\mathrm{LDH})=500 \mathrm{mg} / \mathrm{dl}$, bilirrubina direta $=0,68 \mathrm{mg} / \mathrm{dl}$, bilirrubina indireta $=0,97 \mathrm{mg} / \mathrm{dl}$, hematócrito $(\mathrm{Ht})=21,4 \%$ e hemoglobina $(\mathrm{Hb})=7,3 \mathrm{~g} / \mathrm{dl}$.

O paciente apresentou o ecocardiograma realizado há 1 ano, com átrio esquerdo de $4,3 \mathrm{~cm}$, diâmetro diastólico do ventrículo esquerdo de 5,3 cm e sistólico de 3,2 cm, fração de ejeção de $69 \%$ e prótese aórtica metálica normo funcionante com gradiente sistólico transvalvar médio de $14 \mathrm{mmHg}$.

Diante desses achados, suspendeu-se a varfarina e administraram-se vitamina K endovenosa $10 \mathrm{mg}$, 1 vez ao dia e uma unidade de plasma fresco $(240 \mathrm{ml})$ de 8/8 horas para correção da anticoagulação.

No segundo dia de internação, o paciente mantinha a monoparesia crural de grau 3, porém apresentava-se em melhor estado geral. Exames do dia evidenciaram $\mathrm{Hb}$ de 5,4g/dl, Ht de 16\% e TTPa e RNI iguais a 82,4 e 3,45 segundos, respectivamente. Diante dos achados, mantiveramse a vitamina $\mathrm{K}$ endovenosa e o plasma fresco, e foram administradas 2 unidades de concentrado de hemácias, as quais elevaram $\mathrm{Hb}$ para $8,4 \mathrm{~g} / \mathrm{dl}$ e $\mathrm{Ht}$ para $25 \%$.
Para investigação de possível complicação hemorrágica intra-abdominal, foi solicitada TC de abdome com contraste que evidenciou aumento difuso do volume dos músculos ilíaco e psoas à esquerda, com extensão até sua inserção no trocânter femoral menor esquerdo (Figura 1). No interior do músculo Ilíaco esquerdo, havia coleção de material hiperatenuante, compatível com sangue coagulado, sugerindo presença de hematoma.

Diante do quadro clínico, com alteração sensitiva e motora de membro inferior e presença de hematoma retroperitoneal em sítio dos músculos ilíaco e psoas ipsilaterais, postulou-se o diagnóstico de compressão de plexo femoral.

O paciente evoluiu com alívio completo da parestesia em região pré-tibial, melhora parcial da dor e, ao exame, apresentava força de grau 4 em membro inferior esquerdo. Ante a RNI de 1,83, suspendeu-se o uso de vitamina $\mathrm{K}$ e plasma intravenosos.

No quinto dia de internação, o paciente apresentava $\mathrm{Hb}$ de 10,3 g/dl, Ht de 30,5\%, RNI de 1,95 e força grau de 5 no membro inferior esquerdo. Diante do quadro, foi reiniciado o uso de varfarina na dose de 2,5 mg/dia.

Após 8 dias de internação, o paciente apresentava-se assintomático, com RNI de 2,57 e em condições de alta hospitalar. Recebeu orientação de continuidade de uso de $2,5 \mathrm{mg}$ de varfarina, manutenção do captopril na dose de $25 \mathrm{mg} \mathrm{8/8}$ horas e foi agendado retorno no ambulatório da Clínica Médica para reavaliação de tempo de protrombina e, se necessário, reajuste da dose da varfarina.

No retorno após 7 dias, o paciente negava queixas e apresentava $\mathrm{RNI}$ de 2,55. Foi orientado sobre os cuidados com a anticoagulação e a procurar a emergência do Hospital

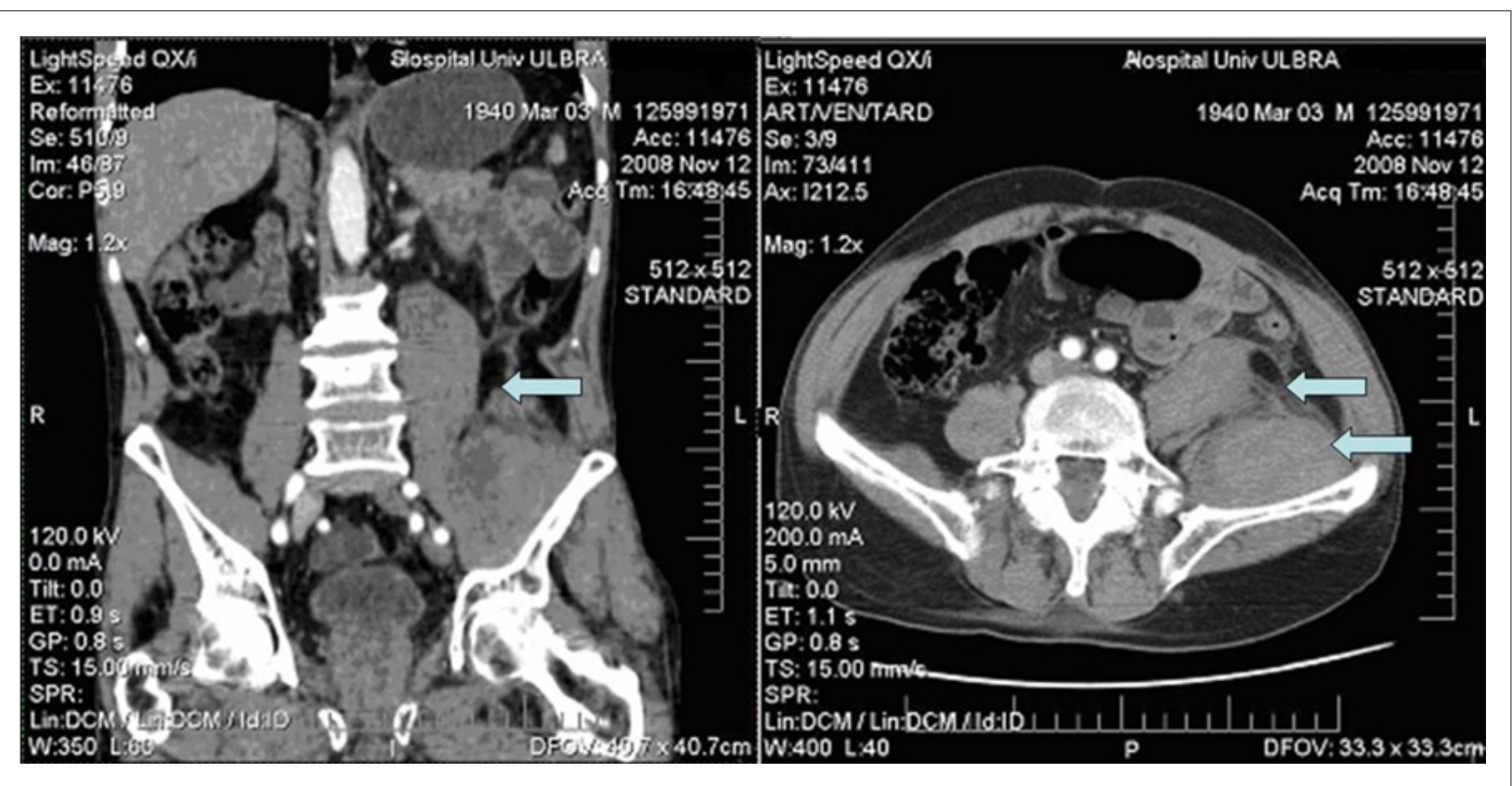

Fig. 1 - Tomografia computadorizada de abdome com contraste evidenciando aumento difuso do volume dos músculos ilíaco e psoas esquerdos, em cortes sagital e transversal. 


\section{Relato de Caso}

Universitário da Ulbra em caso de intercorrências.

\section{Discussão}

Sangramentos e hemorragias são complicações potenciais e graves em pacientes em tratamento anticoagulante, com taxas de até $10 \%$, o que justifica a necessidade de controle rigoroso e regular dos níveis de TP e RNI. São consideradas "hemorragias maiores" aquelas que se desenvolvem, principalmente, em sítio intracraniano ou retroperitoneal e/ou com necessidade de transfusões, internação hospitalar e procedimentos cirúrgicos para compensação ou que levam à morte 3 . Já está estabelecido que pacientes que utilizam varfarina e apresentam elevação dos níveis de TP e RNI, idosos e portadores de doença cerebrovascular e/ou com próteses cardíacas metálicas estão sob maior risco de desenvolvimento de sangramentos ${ }^{4}$. Neste último grupo de pacientes, a prevalência de hemorragia extracraniana é de aproximadamente 2,1\%/ano, com maior tendência no primeiro mês da terapia e queda gradual nos meses seguintes ${ }^{2}$.

A hemorragia retroperitoneal é uma complicação rara, com incidência de $1,3 \%$ a $6,6 \%$ ao ano, podendo ser encontrada em casos de hemofilia, trauma ou anticoagulação $0^{5-7}$. Para compreensão do quadro clínico que acompanha esses pacientes é fundamental o entendimento da anatomia dessa região. O nervo femoral é formado pela união das raízes de L2-L4, as quais percorrem trajeto junto ao tendão do músculo psoas e ilíaco e através do canal femoral, provendo inervação motora e sensitiva ao membro inferior. Por ação compressiva do hematoma, pacientes podem apresentar quadro clínico variável, desde dor lombar ou inguinal súbita, em sua fase inicial (com diagnóstico diferencial incluindo abdome agudo e doenças osteomusculares), até parestesia ou paresia da coxa

\section{Referências}

1. Wysowski DK, Nourjah P, Swartz L. Bleeding complications with warfarin use - a prevalent adverse effect resulting in regulatory action. Arch Intern Med. 2007; 167 (13): 1414-9.

2. Levine MN, Raskob G, Landefeld CS, Kearon C. Hemorrhagic complications of anticoagulant treatment. Chest. 2001; 119 (Suppl. 1): 108S-121S.

3. Wada Y, Yanagihara C, Nishimura Y. Bilateral Iliopsoas hematomas complicating anticoagulant therapy. Internal Medicine (Tokyo). 2005; 44: 641-3.

4. Casais P, Luceros AS, Meschengieser S, Fondevila C, Santarelli MT, Lazzari MA. Bleeding risk factors in chronic oral anticoagulation with acenocoumarol. Am J Hematol. 2000; 63: 192-6.

5. Uncini A, Tonali PL, Falappa P, Danza FM. Femoral neuropathy from iliac muscle hematoma induced by oral anticoagulation therapy. J Neurol. 1981; 226: $137-41$. e perna, ou sangramento maciço e choque $\mathrm{e}^{3,6,8}$. O diagnóstico é baseado nas manifestações clínicas e em exame de imagem (tomografia computadorizada), evidenciando aumento do volume da musculatura comprometida ${ }^{9}$.

Estudos mostram que o risco de sangramento cresce de forma diretamente proporcional à elevação da RNI, sendo significativamente maior quando esta é superior a 3,0 ${ }^{10}$. No caso apresentado, o paciente apresentava RNI superior a 5,0, o que representa risco bastante elevado de complicações hemorrágicas.

O tratamento em casos de neuropatia femoral por hematoma retroperitoneal secundário a esquema de anticoagulação é controverso. Manejo conservador, com descontinuação das medicações e reversão da coagulopatia por meio de administração de vitamina $\mathrm{K}$ e plasma fresco, pode levar à recuperação completa. Quando o hematoma é extenso ou há sintomatologia exuberante, fasciotomia com drenagem do hematoma está indicada ${ }^{7}$.

\section{Potencial Conflito de Interesses}

Declaro não haver conflito de interesses pertinentes.

\section{Fontes de Financiamento}

O presente estudo não teve fontes de financiamento externas.

\section{Vinculação Acadêmica}

Não há vinculação deste estudo a programas de pósgraduação.

6. Holscher RS, Leyten FSS, Oudenhoven LFIJ, Puylaert JBCM. Percutaneous decompression of an iliopsoas hematoma. Abdom Imaging. 1997; 22: 114-6.

7. Parmer SS, Carpenter JP, Fairman RM, Velazquez OC, Mitchell ME. Femoral neuropathy following retroperitoneal hemorrhage: case series and review of the literature. Ann Vasc Surg. 2006; 20: 536-40.

8. Seijo-Martinez M, Castro del Rio M, Fontoira E, Fontoira M. Acute femoral neuropathy secondary to an iliacus muscle hematoma. J Neurol Sci. 2003; 209: 119-22.

9. Lenchiki L, Dovgan DJ, Kier R. CT of the iliopsoas compartment: value in differentiating tumor, abscess and hematoma. Am J Roentgenol. 1994; 162: 83-6.

10. Oake N, Jennings A, Forster AJ, Fergusson D, Doucette S, Walraven CV. Anticoagulation intensity and outcomes among patients prescribed oral anticoagulant therapy: a systematic review and meta-analysis. CMAJ. 2008; 179 (3): 235-44. 